\title{
Medidas psicométricas de la cohesión en equipos de trabajo universitarios Psychometric measures of cohesion in university work teams
}

\author{
Irene Checa Esquiva, María Rocío Bohórquez Gómez-Millán \\ Universidad de Valencia (España), Universidad de Sevilla (España)
}

\begin{abstract}
Resumen. La cohesión ha sido un concepto ampliamente estudiado en equipos deportivos y organizacionales, pero no ha sido estudiado en equipos de trabajo universitarios. Además se han usado medidas de la cohesión, como el Sociograma, que no han demostrado su validez de constructo. Este trabajo presenta dos estudios. En el primero, el objetivo es comprobar las evidencias de validez confirmatoria y fiabilidad del GEQ (Group Environmental Questionnaire) en equipos de trabajo universitarios y en él han participado 273 alumnos. En el segundo estudio, se quieren comparar las medidas de la cohesión a través de dos instrumentos: GEQ y Sociograma utilizando dos mediciones antes y después del trabajo grupal. Esta segunda muestra está compuesta por 72 alumnos agrupados en 4 equipos de tamaño similar a un equipo deportivo. Los resultados demuestran que el GEQ presenta niveles de ajuste buenos en equipos de trabajo universitarios (CFI =.995; TLI =.994; RMSEA =.04) y valores de fiabilidad aceptables. Por otra parte, el segundo estudio demuestra que tras el trabajo grupal, las mediciones de la cohesión a través del GEQ aumentan en sus 4 factores, mientras que la medida cohesión con el Sociograma se mantiene sin cambios. Se concluye que el GEQ es un instrumento sensible a los cambios producidos en la cohesión de un grupo a medida que éste se crea, construye y evoluciona y sin embargo el Índice de Cohesión del Sociograma no lo es, y por tanto no reúne suficientes evidencias de validez de constructo para medir la cohesión.
\end{abstract}

Palabras clave: evaluación, cohesión, sociograma, test psicométrico, estudiantes universitarios.

Abstract. Cohesion has been a widely studied concept in sports and organizational teams but has not been studied in university work teams. In addition, evaluation forms of cohesion have been used, yet their construct validity has not been demonstrated. This work presents two studies. In the first one, the objective is to verify the evidence of confirmatory validity and reliability of the GEQ (Group Environmental Questionnaire) in university work teams through the participation of 273 students. In the second study, we aim to compare the degree of cohesion through both GEQ and Sociograms using two evaluation questionnaires before and after group work. This second sample is composed by 72 students grouped into 4 teams of similar size as that of sports teams. The results show that the GEQ presents good adjustment levels in university work teams $(\mathrm{CFI}=.995, \mathrm{TLI}=.994, \mathrm{RMSEA}=.04)$ and acceptable reliability values. On the other hand, the second study shows that after group work the degree of cohesion through the GEQ increases in its 4 factors, while the extent of cohesion remains unchanged when analyzed through Sociograms. As a conclusion, the GEQ is an instrument sensitive to the changes produced in the cohesion of a group through its creation, construction, and evolution, whilst the Sociogram Cohesion Index is not. Therefore, the latter does not display sufficient evidence of Construct validity to measure cohesion.

Keywords: group, evaluation, university, cohesion, test, sociogram.

\section{Introducción}

La cohesión de los equipos de trabajo es un aspecto fundamental a tener en cuenta a la hora de entender y explicar la participación, la implicación y el rendimiento de los equipos (Bohórquez, Delgado y Fernández, 2017; Iturbide, Elosua, y Yanes, 2010). El concepto se empleó originariamente en el contexto deportivo donde ha sido ampliamente estudiado (Carron, 1980; García-Mas et al., 2006; Whitton \& Fletcher, 2014; Leo, García-Calvo, Parejo, Sánchez \& SánchezOliva, 2010) trasladándose posteriormente a otros contextos como el organizacional (Charbonneau \& Wood, 2018; Urien, Osca \& García-Salmones, 2017) o el educativo (Lafferty, Wakefielf \& Brown, 2016; Martín, Díaz-Aguado, Martínez \& Steglich, 2016).

Aunque tradicionalmente se consideró un concepto unitario (Lewin, 1947), esta idea ha ido progresando hasta llegar a considerarse un constructo multidimensional y dinámico (Yukelson, Weinberg \& Jackson, 1984; Dion, 2000). Carron, Widmeyer, \& Brawley (1998) han aportado la definición más utilizada hasta el momento, considerando la cohesión como un «proceso dinámico que se refleja en la tendencia de un grupo a no separarse y permanecer unido con la finalidad de

\footnotetext{
Fecha recepción: 07-09-18. Fecha de aceptación: 18-07-19 Irene Checa Esquiva
} irene.checa@uv.es conseguir sus objetivos instrumentales y/o para la satisfacción de las necesidades afectivas de sus miembros» (p.214). Esta definición está basada en el Modelo Multidimensional de la Cohesión elaborado por Carron (1982), que tiene en cuenta dos percepciones de los integrantes del equipo: las percepciones individuales de los miembros hacia el grupo en su totalidad (integración) y las atracciones personales de un miembro hacia el grupo (atracción interpersonal). Ambas pueden además orientarse a aspectos relacionados con la tarea o sociales; los relacionados con la tarea tienen que ver con cómo los miembros del equipo se unen para alcanzar objetivos comunes mientras que los que tienen que ver con el aspecto social reflejan la motivación para desarrollar y mantener las relaciones sociales dentro del grupo.

Dada la importancia de la cohesión en los equipos de trabajo de cualquier ámbito, realizar una medición precisa de este constructo es esencial (Salas, Grossman, Hughes \& Coultas, 2015). Uno de los primeros instrumentos que se ha utilizado es el Multidimensional Sport Cohesion Instrument -MSCI-(Yukelson, et al., 1984). Este cuestionario está compuesto por 22 ítems, e incluye cuatro dimensiones generales de la cohesión grupal: atracción hacia el grupo, unidad de propósitos, calidad de trabajo en equipo y roles valorados. De acuerdo con la definición de estos factores, el MSCI hace referencia tanto a la cohesión social como a la cohesión de tarea, aunque se ha diseñado sobre todo para medir este segundo aspecto, representado por las dimensiones de unidad de propósitos, calidad de trabajo en equipo y roles valo- 
rados (García-Quinteiro, Rodríguez-Casal, Andrade, \& Arce, 2006).

Sin embargo, el instrumento más utilizado es el Group Environment Questionnaire -GEQ-, elaborado por Carron, Brawley \& Widmeyer (1998) que, combinando los aspectos individuo-grupo y social-tarea, contiene cuatro factores evaluados en 18 ítems: integración grupal-tarea (GI-T), integración grupal-social (GI-S), atracción interpersonal hacia la tarea del grupo (ATG-T), atracción interpersonal hacia los aspectos sociales del mismo (ATG-S). Debido a algunas críticas sobre el análisis factorial y la consistencia interna de la prueba, diferentes autores redujeron el número de ítems, pero no pudieron confirmar la estructura de cuatro factores (Carron, Bray \& Eys, 2002; Sullivan, Short \& Cramer, 2002). Leo, González-Ponce, Sánchez-Oliva, Pulido \& García-Calvo (2015) realizaron un estudio para la validación y adaptación al español de una versión corta del GEQ con jugadores de fútbol profesionales y supuso la reducción de la versión original a 12 ítems redactados de forma positiva y agrupados dentro de los cuatro factores originales (GI-T, GI-S, ATG-T, ATG-S). Esta versión corta resultó ser un instrumento válido y fiable para medir la cohesión de equipo en ese contexto. A pesar de que el GEQ ha sido empleado como instrumento de evaluación de la cohesión grupal en ámbitos diferentes al deportivo (Ahronson \& Cameron, 2007; Barrutia et al., 2003; GarcíaGuiu, Molero \& Moriano, 2015; García-Guiu, Molero, Moya \& Moriano, 2015, 2016; Knouse, 2007; Paredes, 2013), no existen validaciones adaptadas a equipos de trabajo universitarios.

Por su parte, el Sociograma es una técnica útil para determinar las preferencias de los miembros de un grupo en relación a diferentes estímulos -personas- que forman parte de su medio (Rodríguez, 2001). Tal y como señalan Chelcea (2005), Rodríguez (2001) o Sabin, Mihai \& Marcel (2014) es posible recurrir al sociograma para conocer las relaciones existentes entre los miembros de un grupo, la intensidad de las mismas, la posición de cada miembro en relación a los otros, la estructura informal que presentan como colectivo... y también el grado de cohesión del grupo. Esta idea de que el sociograma puede ser empleado para evaluar la cohesión de un grupo se ve reflejada en la investigación acerca de grupos propios de entornos educativos (Bautista et al., 2009, Llopis \& Llopis, 1998) y de entornos deportivos (Canto y Hernández-Mendo, 2005; Díez \& Márquez, 2005; Llopis \& Llopis, 2001, 2002). El argumento en la base para el empleo del sociograma como medida de la cohesión de un grupo radica en el carácter interaccional de la misma. Según este postulado, y como señalan Canto et al., (2005), el Índice de Cohesión del Sociograma sería válido para la evaluación de la cohesión grupal aunque presentaría ciertas limitaciones derivadas del número de sujetos del grupo y la formalidad del mismo. En el primer caso, dado que cada miembro tiene una capacidad máxima de expansión hacia su grupo, en un equipo altamente cohesionado aparecería un efecto techo a partir del cual el índice se estabilizaría y dejaría de aportar información. En lo relativo a la estructuración de los equipos, grupos altamente jerarquizados mostrarían según el índice sociométrico de cohesión o afiliación puntuaciones significativamente menores que otros con escasa verticalidad en su estructura.
Por todo ello, este trabajo presenta dos estudios con el objetivo de determinar si el sociograma es una herramienta válida para la evaluación de la cohesión grupal en el contexto deportivo. En el Estudio 1 se presentan las evidencias de validez de constructo a través del análisis factorial confirmatorio (AFC), consistencia interna y evidencias de validez convergente del GEQ en una muestra de estudiantes, para comprobar si este instrumento es válido para medir la cohesión en equipos de trabajo universitarios. Por otra parte, en el Estudio 2 se presentan los resultados en la variable cohesión obtenidos a través del GEQ y a través del Índice de Cohesión del sociograma para comprobar su equivalencia o no y discutir su uso.

\section{Estudio 1}

\section{Método}

\section{Participantes}

Participaron en el estudio 273 sujetos, todos ellos entre 18 y 24 años ( $M=20.34$, DT= 2.89) y estudiantes de la Universidad de Sevilla, pertenecientes a diferentes estudios oficiales de Grado (Ciencias de la Actividad Física y el Deporte o Pedagogía) o Postgrado (Master en Recursos Humanos o Máster en Psicopedagogía). En relación al tamaño de los equipos de trabajo empleados como referencia para la evaluación de la cohesión, la mayor parte (96.7\%) pertenecían a un equipo de 4 miembros, mientras que el resto formaban parte de equipos de 3 miembros. Estos equipos tenían como objetivo llevar a cabo los contenidos prácticos de las asignaturas.

\section{Instrumentos}

Datos socio-demográficos. Se preguntó a los participantes por un conjunto de datos socio-demográficos tales como edad, estudios en curso, tiempo de pertenencia al grupo, tamaño del equipo de trabajo y duración esperada del mismo.

Cohesión grupal. Se utilizó una adaptación de la versión española del Group Environment Questionnaire de Leo et al. (2015) al ámbito universitario. El GEQ consta de 12 ítems agrupados en cuatro factores: atracción grupal hacia la tarea, atracción grupal hacia lo social, integración individual en la tarea e integración individual en lo social; estos 4 factores hacen referencia a las orientaciones social y de tarea en sus percepciones de atracción e integración. El formato de respuesta utilizado fue una escala tipo Likert del 1(nada) al 5 (mucho). Se adaptaron 8 de los 12 ítems al contexto universitario y los cambios realizados fueron: «actividades extradeportivas» por «extra-académicos», «juego» por «trabajo», «entrenamientos y partidos» por «sesiones de trabajo», «jugadores» por «miembros» $\mathrm{y}$ «temporada» por «proyecto». En la validación original de la escala presentó valores de consistencia interna todos superiores a .70 y se mostró invariante por género.

Además, con el objetivo de facilitar la obtención de evidencias de validez convergente de la escala, se incluyó el mono-ítem «Siento que mi equipo está cohesionado», manteniendo la escala de respuestas propuesta para el GEQ. 


\section{Procedimiento}

Tras recibir la autorización por parte de la Universidad de Sevilla para realizar este estudio dentro del contexto de las asignaturas correspondientes, todas las personas que conformaron la muestra respondieron al cuestionario de cohesión en su totalidad, en formato de lápiz y papel, al inicio de la jornada académica. Se les pidió que utilizaran su equipo de trabajo en alguna asignatura concreta como referencia para contestar. La confidencialidad y el anonimato de los datos fue asegurado a los participantes y todos ellos consintieron su participación en el estudio y firmaron el documento de consentimiento informado.

\section{Análisis de datos}

En este trabajo se realizaron los pertinentes análisis estadísticos con el software MPlus 7.4 (Muthen \& Muthen, 2017). Teniendo en cuenta que la estructura de 4 factores del GEQ ha sido demostrada en varios estudios (Carron et al., 1985; Leo et al., 2015), se realizaron directamente Análisis Factoriales Confirmatorios. Se pusieron a prueba los modelos confirmatorios de primer orden presentes en la literatura: un modelo unidimensional de Cohesión Global (M1); un modelo con dos factores de primer orden representando a los factores Atracción e Integración (M2); un modelo de cohesión con dos factores de primer orden representando los factores Social y Tarea (M3); un modelo de cohesión con cuatro factores de primer orden, tal y como fue propuesto por Carron et al., (1985).

Para el análisis, se utilizó el estimador WLSMV (siglas en inglés de estimador robusto de mínimos cuadrados ponderados), que es apropiado para el AFC con datos categóricos. Este análisis se realiza sobre la matriz de correlaciones policóricas. Los índices de ajuste utilizados fueron $x^{2}$, comparative fit index (CFI), Tucker-Lewis index (TLI), y root mean square error of approximation (RMSEA). En cuanto a los valores cuantitativos de referencia, valores de CFI y TLI > .95 y RMSEA < .06 son considerados indicadores de un ajuste excelente (Bentler, 2007) y valores de CFI y TLI > .90 y RMSEA < .08 son considerados aceptables (Marsh, Hau y Wen, 2004).

Se llevó a cabo el cálculo de la consistencia interna de la escala a través de los valores de $\alpha$ de Cronbach y se obtuvieron evidencias de validez convergente utilizando la correlación de la puntuación total de la escala con una medida mono-ítem de cohesión.

\begin{tabular}{|c|c|c|c|c|c|c|c|c|c|}
\hline \multicolumn{10}{|c|}{ Porcentajes de respuesta, asimetría y curtosis de cada ítem del GEQ } \\
\hline Ítems & \%Resp.1 & \%Resp.2 & \%Resp.3 & \multicolumn{2}{|c|}{ \%Resp.4 } & \multicolumn{2}{|l|}{ \%Resp.5 } & \multirow{2}{*}{$\begin{array}{l}\text { Asim } \\
-.913\end{array}$} & \multirow{2}{*}{$\begin{array}{l}\text { Curt. } \\
.097\end{array}$} \\
\hline GEQ1 & 1.1 & 5.1 & 19.0 & \multicolumn{2}{|l|}{28.2} & 46.5 & -.913 & & \\
\hline GEQ2 & 0.7 & 2.2 & 1.6 & \multicolumn{2}{|l|}{51.6} & 34.8 & \multicolumn{2}{|r|}{-1.013} & 1.865 \\
\hline GEQ3 & 3.3 & 6.2 & 15.0 & \multicolumn{2}{|l|}{31.5} & 44.0 & \multicolumn{2}{|c|}{-1.106} & .609 \\
\hline GEQ4 & 0.4 & 0.4 & 17.9 & \multicolumn{2}{|l|}{44.7} & 36.6 & \multicolumn{2}{|r|}{-.549} & .030 \\
\hline GEQ5 & 14.3 & 22.0 & 34.4 & \multicolumn{2}{|l|}{19.4} & 9.9 & \multicolumn{2}{|r|}{.057} & -.738 \\
\hline GEQ6 & 0 & 4.8 & 22.7 & \multicolumn{2}{|l|}{44.7} & 27.8 & \multirow{2}{*}{\multicolumn{2}{|c|}{-.414}} & -.459 \\
\hline GEQ7 & 5.5 & 15.0 & 26.0 & \multicolumn{2}{|l|}{25.6} & 27.8 & -.384 & & -.823 \\
\hline GEQ8 & 0.4 & 1.5 & 11.0 & \multicolumn{2}{|l|}{37.7} & 49.5 & & -1.074 & 1.110 \\
\hline GEQ9 & 4.0 & 6.2 & 19.8 & \multicolumn{2}{|l|}{32.6} & 37.4 & \multicolumn{2}{|c|}{-.915} & .248 \\
\hline GEQ10 & 0.4 & 1.8 & 15.8 & \multicolumn{2}{|l|}{35.2} & 46.9 & & -.888 & .298 \\
\hline GEQ11 & 2.6 & 8.1 & 21.2 & \multicolumn{2}{|l|}{28.9} & 39.2 & \multicolumn{2}{|r|}{-.774} & -.186 \\
\hline GEQ12 & 0 & 1.8 & 8.1 & \multicolumn{2}{|l|}{43.2} & 46.9 & \multicolumn{2}{|r|}{-.936} & .723 \\
\hline \multicolumn{10}{|c|}{$\begin{array}{l}\text { Tabla } 2 \\
\text { Modelos factorigles confirmatorios }\end{array}$} \\
\hline \multicolumn{3}{|c|}{ Modelo } & $?^{2}$ & \multicolumn{2}{|l|}{ gl } & & ILI & \multicolumn{2}{|c|}{ RMSEA } \\
\hline M1 1F C & esión globa & & 586.546 & 54 & .923 & & .906 & .19 & \\
\hline $\mathrm{M} 22 \mathrm{FAt}$ & cción e Inte & gración & 537.500 & 53. & .930 & & .913 & .18 & \\
\hline M3 2F So & al y Tarea & & 181.477 & 53 & .981 & & 977 & .09 & \\
\hline M4 $4 \mathrm{Fac}$ & & & 79.683 & 48 & .995 & & 994 & .04 & \\
\hline
\end{tabular}

\section{Resultados}

Los datos descriptivos de los ítems se muestran en la Tabla 1 con los porcentajes de respuesta, la asimetría y los valores de curtosis. Como se puede observar, ningún participante respondió «nada» a los ítems 6 y 12, todos los ítems presentan una asimetría negativa (excepto el ítem 5) y los valores de curtosis se encuentran todos entre el intervalo aceptable de $-4 /+4$.

Los resultados de ajuste de los modelos puestos a prueba se muestran en la Tabla 2. Como puede observarse, el modelo unidimensional (M1) obtiene resultados muy pobres, de la misma forma que el modelo de $2 \mathrm{~F}$ que separa entre Atracción e Integración, que obtiene CFI=.930 y RMSEA muy alto. Sin embargo, el modelo de $2 \mathrm{~F}$ que presenta factor Social y Tarea ya obtiene índices de ajuste aceptables, mientras que el valor residual sigue estando por encima de valores aceptables (RMSEA=-.09). Finalmente, el modelo de cuatro factores de primer orden es el que mejor ajuste obtiene en todos los índices propuestos.

En cuanto a la fiabilidad, los valores de á de Cronbach son excelentes para el factor Integración Social $(\alpha=.91)$, buenos para el factor Atracción Social $(\alpha=.80)$ y para el factor Integración Tarea $(\alpha=.75)$, mientras que para el factor Atracción Tarea, se obtienen los resultados pobres en cuanto a la consistencia interna ( $\alpha=$.65). Por otro lado, se obtuvieron evidencias de validez convergente a través de la correlación con la medida mono-ítem de cohesión. En todos los factores los valores fueron aceptables con $r=.622(p<.01)$ para el factor Atracción Social, $r=.611(p<.01)$ para el factor Atracción Tarea, $r=.568(p<.01)$ para el factor Integración Tarea y $r=.593(p<.01)$ para el factor Integración Social.

\section{Estudio 2}

Las hipótesis planteadas para el estudio 2 son:

1) Las puntuaciones en los 4 factores del GEQ (Integración Social, Atracción Social, Integración Tarea, Atracción Tarea) se incrementarán entre las mediciones pre y post del trabajo de equipo.

2) El Índice de Asociación o Cohesión del sociograma no variará entre las mediciones pre y post del trabajo de equipo.

\section{Método}

\section{Participantes}

Participaron en el estudio 72 sujetos, todos ellos comprendidos entre los 18 y 34 años de edad (M= 21.03, DT= 3.06). Los participantes eran estudiantes de la Universidad de Sevilla pertenecientes al Grado de Ciencias de la Actividad Física y el Deporte y cursando la asignatura Psicología Social del Deporte. Se agruparon en 4 equipos estables desde 14 hasta 20 miembros.

\section{Instrumentos}

Para este estudio se utilizó el instrumento GEQ, que ya ha sido descrito en el Estudio 1, y que presenta en esta muestra valores de consistencia interna aceptables en los 4 factores (á= .82-.70).

Para calcular el Î́ndice de Asociación o Cohesión se utilizó el Sociograma (Moreno, 1954), que mide y representa las 
relaciones interpersonales dentro de un grupo determinado. Se trata de una técnica que determina las preferencias de los individuos respecto al resto de personas de su grupo (Rodríguez, 2001). Independientemente del sistema de creación de los equipos deportivos, el sociograma es una técnica útil para determinar cómo los miembros de un equipo se relacionan entre sí. Para este trabajo se diseñaron 4 preguntas para reflejar tanto las relaciones formales como informales presentes en la estructura grupal: (a) ¿A cuál de tus compañero/s de equipo preferirías como capitán?; (b) ¿Qué compañero/s preferirías que no fuera capitán?; (c) ¿Con qué compañero/s de tu equipo saldrías después de la clase a tomar algo?; (d) ¿Con qué compañero/s de tu equipo no sueles salir después de la clase a tomar algo? En todas las cuestiones era necesario nombrar al menos a uno de los compañeros de equipo y el número máximo de respuestas se limitó a seis.

Además del GEQ y el Sociograma los estudiantes contestaron a un cuestionario de datos sociodemográficos que incluía la edad, el sexo, el nombre del grupo y el tamaño del mismo.

\section{Procedimiento}

El desarrollo de este trabajo fue llevado a cabo dentro de la asignatura de Psicología Social del Deporte del Grado de Ciencias del Deporte en la Universidad de Sevilla, correspondiente al segundo cuatrimestre del curso (febrero-junio). Para realizar la parte práctica de esta asignatura, las actividades están diseñadas para realizarse en grupos equivalentes a un equipo de deporte colectivo (fútbol, baloncesto, balonmano, rugby...). De esta forma, los grupos fueron creados forzadamente para que estuvieran compuestos por un mínimo de 14 y un máximo de 20 estudiantes. Las 15 semanas del cuatrimestre constituyen una liga deportiva en la que los equipos formados se enfrentan dos a dos, puntuando en dos categorías diferenciadas: deportiva por un lado y de desempeño de competencias psicosociales por otro. Las semanas 2 a 8 (ambas inclusive) constituían la primera vuelta de liga, la jornada 9 se desarrollaba un mercado de invierno y las jornadas 10 a 15 (ambas inclusive), constituían la segunda vuelta de la liga. Este procedimiento permitía promocionar el aprendizaje cooperativo en situaciones prácticas y, además, desarrollar la cohesión de los equipos (Fernández-Río, 2017).

El primer día de prácticas de la asignatura los estudiantes, que ya se conocían entre sí por haber compartido aula durante todo el primer cuatrimestre, contestaron a los instrumentos propuestos de forma que pudieran ser identificados con un código que asegurara su anonimato. Dos meses después, cuando los grupos ya habían realizado ocho prácticas juntos como equipo, contestaron de nuevo al GEQ y al Sociograma empleando el mismo código que la primera vez para facilitar la identificación entre los dos momentos de evaluación. Los alumnos fueron informados que los datos extraídos serían utilizados para el desarrollo de la asignatura y para fines científicos, y ofrecieron su consentimiento informado.

\section{Análisis de datos}

Los datos extraídos de ambas mediciones fueron analiza- dos con SPSS 24.0 (IBM, 2016). En primer lugar, fueron calculadas las puntuaciones de cada sujeto en los 4 factores del GEQ en el pre y el post-test. Posteriormente, se calculó la media de todo el equipo en esos factores en ambas mediciones, de forma que se obtuviera una puntuación total en cada factor para todo el equipo.

Para extraer el Índice de Asociación o Cohesión del Sociograma se ha calculado dividiendo el número total de elecciones recíprocas entre el número posible de elecciones recíprocas (Chelcea, 2005; Visauta, 1989).

Para analizar si los cambios producidos en los factores del GEQ eran estadísticamente significativos entre el pre y el post se realizaron 4 análisis de varianza (ANOVA) de medidas repetidas separadamente para cada factor del GEQ (Integración Social, Atracción Social, Integración Tarea, Atracción Tarea). En primer lugar, se ha contrastado la hipótesis de igualdad a través de la prueba multivariante Lamba de Wilks. En caso de no existir esfericidad se ha considerado la corrección de Greenhouse-Geisser. Para calcular el tamaño del efecto se presenta el eta cuadrado parcial ( 12 ) que, de acuerdo con los criterios de Cohen (1992), indica un tamaño del efecto pequeño con un $\eta 2$ de .01, moderado con .06 y grande para.14.

Para analizar los cambios producidos en las 4 preguntas del sociograma se han comparado los Índices de Asociación pre y post de cada grupo y se han llevado a cabo 4 pruebas no paramétricas $W$ de Wilcoxon teniendo en cuenta que únicamente se disponía de un dato para todo el grupo, al contrario que en los cálculos de las diferencias pre y post del GEQ. Por ello, y teniendo en cuenta la importancia de los datos intra-grupo, se presentan en forma de figura.

\section{Resultados}

La primera hipótesis planteaba que existirían incrementos en las puntuaciones correspondientes a los cuatro factores del GEQ. Después de comprobar el rechazo de la hipótesis nula de igualdad de medias con la prueba multivariante Lamba de Wiilks, se asumió la ausencia de esfericidad ya que las $4 \mathrm{~W}$ de Mauchly no eran significativas. Por ello, se usó la corrección de Greenhouse-Geisser en los resultados presentados en la Tabla 3. Se observa un aumento en todos los factores del GEQ con valores significativos $(p<.001)$ y un tamaño del efecto grande en todos ellos.

\begin{tabular}{|c|c|c|c|c|c|}
\hline \multicolumn{6}{|l|}{$\begin{array}{l}\text { Tabla } 3 \\
\text { Datos de }\end{array}$} \\
\hline & & Media & DT & $F_{(1,70)}$ & $\eta 2$ \\
\hline \multirow{2}{*}{ AGS } & Pre & 9.15 & .64 & $40.35^{* *}$ & .366 \\
\hline & Post & 10.31 & 1.09 & & \\
\hline \multirow[t]{2}{*}{ AGT } & Pre & 9.22 & .96 & $84.24 * *$ & .548 \\
\hline & Post & 11.42 & 1.21 & & \\
\hline \multirow[t]{2}{*}{ IGT } & Pre & 9.77 & .68 & $24.54^{* *}$ & .260 \\
\hline & Post & 11.02 & 1.55 & & \\
\hline \multirow[t]{2}{*}{ IGS } & Pre & 9.88 & .84 & $41.75^{* *}$ & .374 \\
\hline & Post & 11.49 & 1.41 & & \\
\hline
\end{tabular}

En segundo lugar se hipotetizó que los valores del Índice de Cohesión del sociograma no sufrirían variaciones significativas con el paso de las sesiones prácticas de trabajo. Los resultados del Sociograma demuestran que no existen diferencias entre la medición pre y post en ninguna de las 4 cuestiones: aceptación tarea $(\mathrm{Z}=-.447 ; p=.655)$, rechazo tarea 
( $\mathrm{Z}=-1.00 ; p=.317)$, aceptación social ( $\mathrm{Z}=-1.09 ; p=.273)$ y rechazo social $(\mathrm{Z}=-1.34 ; p=.180)$. En la Figura 1 se observan los mínimos cambios producidos entre las elecciones recípro-
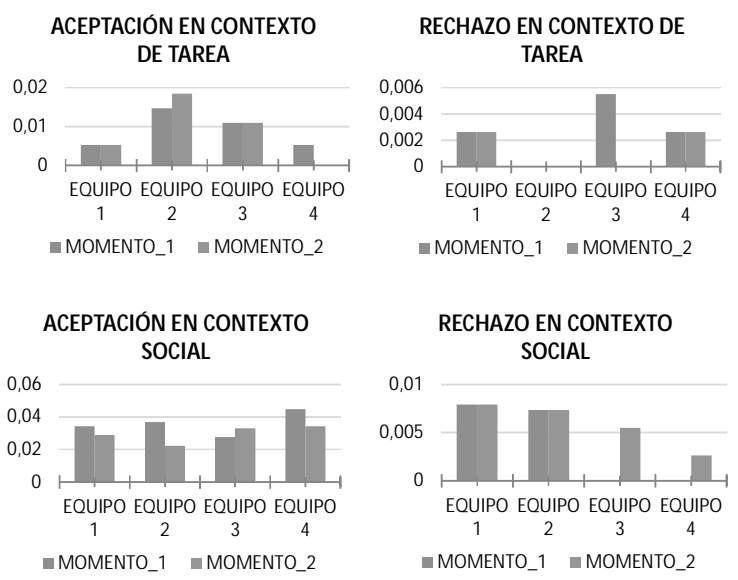

Figura 1

Índices de cohesión de los diferentes equipos en los Momentos 1 y 2 de medición

cas.

\section{Discusión}

El objetivo general de este estudio fue revisar y validar las medidas de cohesión grupal empleadas en contextos educativos y para ello se han desarrollado dos estudios diferentes. El Estudio 1 pretendía comprobar la validez del GEQ en su versión reducida (Leo et al., 2015) en el contexto de los equipos de trabajo en Educación Superior, mientras que el Estudio 2 por su parte perseguía comprobar la equivalencia del GEQ y el Índice de Cohesión del Sociograma como medidas de la cohesión de estos equipos de trabajo.

Puesto que el GEQ había sido empleado en diferentes contextos tanto deportivos como extra deportivos, pero no en el de la educación superior, se procedió a su validación. Aí, los resultados del Estudio 1 indican unas propiedades psicométricas satisfactorias, demostrando una estructura factorial apropiada, consistencia interna buena y evidencias de validez convergente del uso del GEQ en este contexto de los grupos de trabajo universitarios. Partiendo del modelo original de 4 factores (Carron, 1982), los resultados de este trabajo han mostrado una concordancia con ese modelo que separa entre integración tarea y social y atracción tarea y social, de la misma forma que las investigaciones de Carron et al. (1985), Leo et al. (2015) y García-Calvo (2016).

En lo relativo al Estudio 2, se hipotetizó que existirían incrementos pre-post en las puntuaciones correspondientes a los cuatro factores del GEQ y los resultados confirmaron la hipótesis. Los procesos de construcción de equipo o Team Building como los reproducidos en las sesiones prácticas de la asignatura Psicología Social del Deporte a la que pertenecía el alumnado participante en el Estudio 2 generan incrementos significativos en la cohesión del equipo, tal y como ha sido ampliamente demostrado en los contextos deportivos con diferentes experiencias aplicadas y de laboratorio (Bohórquez, Lorenzo, Bueno \& Garrido, 2012; Chicau, Silva \& Palmi, 2012; Lafferty et al., 2016; Stewart, Carreau \& Bruner, 2016) y educativo (Mathieu, Kukenberger, D’Inocenzo \& Reilly, 2015).

Además, se hipotetizó que los valores del Índice de Co- hesión del Sociograma no sufrirían variaciones con el paso de las sesiones prácticas de trabajo. Los resultados del Sociograma no arrojaron diferencias entre las mediciones pre y post en ninguna de las 4 cuestiones (aceptación tarea, rechazo tarea, aceptación social y rechazo social). Los datos arrojados por el índice de Cohesión de los diferentes equipos no permiten concluir que la falta de variación sea producto de la aparición de un efecto techo por elevada cohesión o de una estructura vertical, casos señalados por Canto y Hernández-Mendo (2015) como de limitada eficacia de este índice para la evaluación de la cohesión grupal.

Si bien no se ha encontrado investigación previa que compare los resultados del Índice de Cohesión del Sociograma en dos momentos diferentes para un mismo equipo, la teoría acerca de los grupos permite explicar esta ausencia de cambios. Si las relaciones sociales son en sí formas encasilladas de interacción social mediante patrones establecidos y estables que duran lo suficiente para volverse partes identificables de una estructura social (Merton, 1957; Rodríguez, 2001), entonces es esperable que el sociograma como instrumento que refleja dichas relaciones no cambie de forma sustancial con el mero paso del tiempo.

Aquellos trabajos que emplearon el Sociograma como instrumento para evaluar la cohesión de un grupo (Bautista et al., 2009, Canto et al., 2005; Díez et al., 2005; Llopis et al.,, 1998, 2001, 2002; Sabin et al., 2014) destacan que el empleo del sociograma como medida de la cohesión de un grupo radica en el carácter interaccional de la misma. Sin embargo, tal y como señalan Sabin et al., (2005), un determinado grupo puede tener una elevada densidad de elecciones y rechazos (es decir, interacciones) entre sus miembros, pero tener un Índice de Cohesión bajo e incluso negativo como ocurre en los resultados obtenidos en este estudio. Por lo tanto, además de las limitaciones del sociograma para evaluar la cohesión de un grupo ya señaladas por Canto et al., (2005) como verse influenciado por el tamaño del equipo, el nivel de formalidad del mismo, o la composición de su estructura; habría que sumar la diferenciación conceptual entre patrón de relaciones interpersonales y tendencia de los miembros del grupo a permanecer unidos hasta la consecución de su objetivo (Carron, 1982).

Este trabajo presenta algunas limitaciones. En el Estudio 1, no se preguntó a los participantes por algunos datos sociodemográficos como el sexo, dato que podría haber sido útil para asegurar la invarianza del modelo. En el Estudio 2 las medidas se tomaron al inicio de las clases prácticas y a mitad del periodo de clases, a pesar de que el alumnado se conocía antes del inicio de la asignatura y por lo tanto sus patrones de interacción estaban pre-establecidos. Un tiempo previo de construcción de procesos grupales como identidad o normas podría haber maximizado los resultados encontrados.

Finalmente, es posible concluir que: (a) la versión reducida del GEQ (Leo et al., 2015) es un instrumento válido y fiable para la medida de la cohesión grupal en equipos de trabajo universitarios; y (b) mientras el GEQ es un instrumento sensible a los cambios producidos en la cohesión de un grupo a medida que éste se crea, construye y evoluciona, el Índice de Cohesión del Sociograma no lo es, y por tanto no reúne suficientes evidencias de validez de constructo para medir la 
cohesión.

\section{Referencias}

Ahronson, A., \& Cameron, J. E. (2007). The nature and consequences of group cohesion in a military sample. Military Psychology, 19, 9-25. http://dx.doi.org/10.1080/ 08995600701323277

Barrutia, I., Beitia, l., González, B., Leza, N., Sagasta, A., Martínez, S., Arcaute,...Gallardo, M. (2003) Aportaciones metodológicas a la cohesión de equipos sometidos a desgaste emocional. Trastornos Adictivos, 5(2), 11429. https://doi.org/10.1016/S1575-0973(03)78560-2

Bautista, E., Casas, E., Pineda, I., Bezanilla, J. M., Rebero, L., \& Silva, Y. (2009). Utilidad del sociograma como herramienta para el análisis de las interacciones grupales. $P$ sicología para América Latina, 18(nov), 0-0.

Bentler, P. M. (2007). On tests and indices for evaluating structural models. Personality and Individual differences, 42(5), 825-829. https://doi.org/10.1016/ j.paid.2006.09.024

Bohórquez, M. R., Delgado, P., Fernández, J. (2017). Rendimientos auto y hetero-percibidos y cohesion grupal: un studio piloto. Retos, 31, 103-106.

Bohórquez, M. R., Lorenzo, M., Bueno, M. R., \& Garrido, M: A. (2012). Influencia de la identidad grupal en la cohesión: estudio piloto. Cuadernos de Psicología del Deporte, 12(1), 151-154.

Canto, J. M., Hernández-Mendo, A. (2005). La cohesión en los grupos deportivos. En A. Hernández-Mendo (Coord.), Psicología del Deporte (Vol I.). Sevilla: Wanceulen.

Carron, A. V. (1980). Social Psychology of Sport. Ithaca, NY: Mouvement.

Carron, A. V. (1982). Cohesiveness in Sport Groups: Interpretations and Considerations. Journal of Sport Psychology, 4, 123-138. http://dx.doi.org/10.1123/ jsp.4.2.123

Carron, A. V., Brawley, R. L., \& Widmeyer, W. N. (1998). The measurement of cohesiveness in sport groups. En J. L. Duda (Ed.), Advances in Sport and Exercise Psychology Measurement, (pp. 213-226). Ithaca, NY: Fitness Information Technology.

Carron, A. V., Bray, S. R., \& Eys, M. A. (2002). Team cohesion and team success in sport. Journal of Sport Sciences, 20, 119-126. http://dx.doi.org/10.1080/ 026404102317200828

Carron, A. V., Widmeyer, W. N., \& Brawley, L. (1985). The Development of an Instrument to Assess Cohesion in Sport Teams: The Group Environment Questionnaire. Journal of Sport Psychology, 7(3), 244-266. Tomado de: http://ess22012.files.wordpress.com/2012/10/geq.pdf

Charbonneau, D., \& Wood, V. (2018). Antecedents and outcomes of unit cohesion and affective commitment to the Army. Military Psychology, 30(1), 43-53. http:// doi.org/10.1080/08995605.2017.1420974

Chelcea, S. (2005). Social Psychology. Bucharest: Ed. Economicã.

Chicau, C., Silva, C., \& Palmi, J. (2012). Programa de Intervención psicológica para la optimización del concepto de equipo (team building) en jóvenes futbolistas. Revista de Psicología del Deporte, 21(1), 49-58.
Cohen, J. (1992). A power primer. Psychological bulletin, 112(1), 155.

Díez, A. \& Márquez, S. (2005). Utilización de sociogramas para la valoración de la cohesión interna de los jugadores de un club de fútbol. Motricidad, European Journal of Human Movement, 14(dic), 37-52.

Dion, K. L. (2000). Group cohesion: From «field of forces» to multidimensional construct. Group Dynamics: Theory, Research, and Practice, 4(1), 7-26. http://doi.org/ 10.1037//1089-2699.4.1.7

Fernández-Río, J. (2017). El Ciclo del Aprendizaje Cooperativo: una guía para implementar de manera efectiva el aprendizaje cooperativo en educación física. Retos, 32, 264269.

García-Calvo, T. (2006). Motivación y comportamientos adaptativos en jóvenes futbolistas. University of Extremadura.

García-Guiu, C. Molero, F., \& Moriano, J.A. (2015). Authentic leadership and its influence on group cohesion and organizational identification: the role of organizational justice as a mediating variable. Revista de Psicología Social, 30(1), 60-88. https://doi.org/10.1080/ 02134748.2014.987539

García-Guiu, C., Molero, F., Moya, M., \& Moriano, J.A.(2015). Authentic leadership, group cohesion and group identification in security and emergency teams. Psicothema, 27(1), 59-64. Tomado de http:// www.psicothema.com/psicothema.asp?id=4236

García-Guiu, C., Molero, F., Moya, M., \& Moriano, J.A. (2016). Transformational leadership and group potency in small military units: The mediating role of group identification and cohesion. Journal of Work and Organizational Psychology, 32(3), 145-152. https://doi.org/10.1016/ j.rpto.2016.06.002

García-Mas, A., Olmedilla,A., Morilla, M., Rivas, C., Quinteiro, E. G., \& Toro, E. O. (2006). Un nuevo modelo de cooperación deportiva y su evaluación mediante un cuestionario. Psicothema, 18(3), 425-432. Tomado de: http:// ezproxy.lib.ucf.edu/login? URL=http:// $\mathrm{s}$ e a r c h. e b s c o h o s t . c o m / login.aspx?direct=trueydb=psyhyAN=2006-12461015ysite=ehost-livelnalex.garcia@uib.es

García Quinteiro, E. M., Rodríguez Casal, M., Andrade, E. M., \& Arce, C. (2006). Adaptación del cuestionario MSCI para la medida de la cohesión en futbolistas jóvenes españoles. Psicothema, 18(3), 668-672.

IBM Corp. Released 2016. IBM SPSS Statistics for Windows, Version 24.0. Armonk, NY: IBM Corp

Iturbide, L. M., Elosua, P., \& Yanes, F. (2010). Medida de la cohesión en equipos deportivos. Adaptación al Español del Group Environment Questionnaire (GEQ). Psicothema, 22(3), 482-488. Tomado de: http:// www.redalyc.org/pdf/727/72714400020.pdf

Knouse, S.B. (2007). Building task cohesion to bring teams together. Quality Progress, 40(3), 49-53.

Lafferty, M. E., Wakefielf, C., \& Brown,H. (2016). «We do it for the team»- Student-athletes' initiation practices and their impact on the group cohesion. International Journal of Sport and Exercise Psychology, 15(4), 438446. https://doi.org/10.1080/1612197X.2015.1121507 
Leo, F.M, García-Calvo, T., Parejo, I., Sánchez, P., \& SánchezOliva, D. (2010) Interacción de la cohesión percibida, las expectativas de éxito y el rendimiento en equipos de baloncesto. Revista de Psicología del Deporte, 19(1), 89102.

Leo, F. M., González-Ponce, I., Sánchez-Oliva, D., Pulido, J. J., \& García-Calvo, T. (2015). Adaptation and validation in Spanish of the group environment questionnaire (GEQ) with professional football players. Psicothema, 27(3), 261-268. http://doi.org/10.7334/psicothema2014.247

Lewin, K. (1947). Frontiers in group dynamics II. Channels of group life; social planning and action research. Human relations, 1(2), 143-153. http://doi: 10.1177/ 001872674700100201

Llopis D. \& Llopis R. (1998, septiembre). Socialización Educativa y estatus sociométrico. Un estudio del cambio en las relaciones grupales. Comunicación presentada en el VI Congreso Español de Sociología. A Coruña, España.

Llopis D. \& Llopis R. (2001, septiembre). Estilos educativos y relaciones grupales. Comunicación presentada en el VII Congreso Español de Sociología. Salamanca, España.

Llopis, D., \& Llopis, R. (2002, marzo). La cohesión grupal de un equipo de baloncesto. Evaluación de una experiencia. Comunicación presentada en el II Congreso de Ciencias del Deporte. Madrid, España.

Martín, J., Díaz-Aguado, M. J., Martínez, R., \& Steglich, C. (2017). Power Structure in the Peer Group: The Role of Classroom Cohesion and Hierarchy in Peer Acceptance and Rejection of Victimized and Aggressive Students. The Journal of Early Adolescence 37(9), 1197-1220. https://doi.org/10.1177/0272431616648451

Mathieu, J. E., Kukenberger, M. R., D’Innocenzo, L., \& Reilly, G. (2015). Modeling reciprocal team cohesion-performance relationships, as impacted by shared leadership and members' competence. Journal of Applied Psychology, 100(3), 713-734.

Marsh, H.W., Hau, K.T., \& Wen, Z. (2004). In search of golden rules: Comment on hypothesis-testing approaches to setting cutoff values for fit indexes and dangers in overgeneralizing hu and Bentler's (1999) findings. Structural Equation Modeling, 11(3), pp. 320-341.

Merton, R. (1957). Social Theory and Social Structure. New York: Free Press.

Moreno J. L. (1954). Fundamentos de la Sociometría. Bue- nos Aires: Paidós.

Muthén, L.K. \& Muthén, B.O. (2017). Mplus User's Guide. Eighth Edition. Los Angeles, CA: Muthén \& Muthén

Paredes, M. (2013). Cohesión de equipos de trabajo y clima laboral percibido por los empleados de la universidad de Montemorelos. Apuntes Universitarios, 2(2), 39-56.

Rodríguez, A. (2001). El Sociograma, una técnica útil para representar las relaciones informales. En Rodríguez y Morera (Eds.) El sociograma. Estudio de las relaciones informales en las organizaciones (pp 43-51). Madrid: Pirámide.

Sabin, S. I., Mihai, S., \& Marcel, P. (2014). The Importance and Utility of the Sociometric Survey Method in Physical Education Research. Procedia. Social and Behavioral Sciences, 117, 185-192.

Salas, E., Grossman, R., Hughes, A. M., \& Coultas, C. W. (2015). Measuring Team Cohesion Observations from the Science. Human Factors: The Journal of the Human Factors and Ergonomics Society, 57(3), 365-374. http:/ /doi.org/10.1177/0018720815578267

Stewart, K. L., Carreau. J. M., \& Bruner, M. W. (2016). Team Building Using a Challenge Ropes Course Experience in Youth Sport. Revue phénEPS / PHEnex Journal, 7(3), 43-58.

Sullivan, P. J., Short, S. E., \& Cramer, K. M. (2002). Confirmatory factor analysis of the group environment questionnaire with co-acting sports. Perceptual and Motor Skills, 94(1), 341-347. http://doi.org/10.2466/ pms.2002.94.1.341

Urien, B., Osca, A., \& García-Salmones, L. (2017). Role ambiguity, group cohesion and job satisfaction: A Demands-Resources Model (JD-R) Study from Mexico and Spain. Revista Latinoamericana de Psicología, 49(2), 137-145. http://dx.doi.org/10.1016/j.rlp.2015.09.014 Visauta, B. (1989) La sociometría. En B. Visauta, Técnicas de investigación social. I Recogida de datos (pp.327-358). Barcelona: PPU.

Whitton, S.M., \& Fletcher, R.B. (2014). The Group Environment Questionnaire: A multilevel confirmatory factor analysis. Small Group Research, 45, 68-88. http:/ /doi:10.1177/1046496413511121

Yukelson, D., Weinberg, R., \& Jackson, A. (1984). A multidimensional group cohesion instrument for intercollegiate basketball teams. Journal of Sport Psychology, 6, 103107. https://doi.org/10.1123/jsp.6.1.103

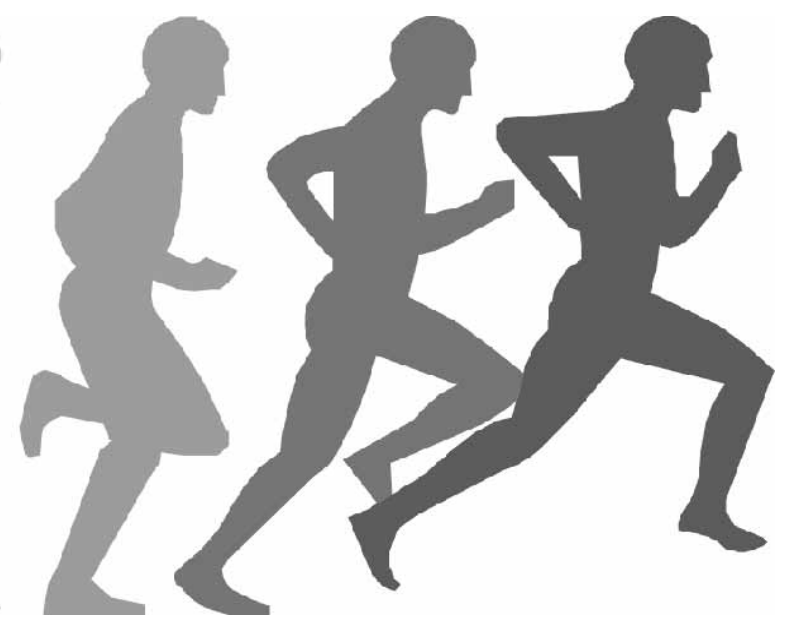

